\title{
ASSESSMENT OF EPIDEMIOLOGY IN PATIENTS WITH MAXILLOFACIAL TRAUMA FROM A SINGLE HOSPITAL OF TABRIZ
}

\author{
Farrokh Farhadi ${ }^{1}$, Mehdi Parvash ${ }^{2}$, Ali Zarandi ${ }^{3}$
}

${ }^{1}$ Assistant Professor, Department of Oral and Maxillofacial Surgery, Faculty of Dentistry, Tabriz University of Medical Sciences, Tabriz, Iran.

2 Doctor Dentist, Faculty of Dentistry, Tabriz University of Medical Sciences, Tabriz, Iran.

${ }_{3}^{3}$ Assistant Professor, Department of Periodontics, Faculty of Dentistry, Tabriz University of Medical Sciences, Tabriz, Iran.

\begin{abstract}
The aim of study was the assessment of epidemiology in patients with maxillofacial trauma, which referring to Emam Reza Hospital of Tabriz, Iran.

\section{MATERIALS AND METHODS}

The study designed as a cross sectional descriptive study and conducted during the one year from 2013-2014. Totally, 250 patients with maxillofacial trauma were selected which referring to Emam Reza Hospital. The demographic data, type of fracture, location of fracture were evaluated and documented in each patient. Data are showed as frequency and analysed by chi-square test by SPSS.21.
\end{abstract}

\section{RESULTS}

$32 \%$ of patients were in the $21-30$ age group and $10.2 \%$ were in the 5 th decade of life. Most fractures were taking place in the subcondylar area (38.28\%). Causes of trauma were 53.9\% accidents, $24.2 \%$ falling, $4.7 \%$ IPV, $11.7 \%$ blunt trauma and $4.7 \%$ sportrelated accidents. Accidents, falling and blunt trauma were the three major causes of fracture in males and females. In females, there was no report of IPV and sport-related accidents. In all age groups, accidents and falling were major aetiologic factors except for blunt trauma being the 2 nd major cause of fracture under 20 years old. Sport-related accidents occurred only in patients under 30 years old.

\section{CONCLUSION}

Most fractures happened in males in 3rd decade of life and in the subcondylar area. Accidents were the major cause of mandibular fractures.

\section{KEYWORDS}

Accident; Fractures; Trauma; Lower Face.

HOW TO CITE THIS ARTICLE: Farhadi F, Parvash M, Zarandi A. Assessment of epidemiology in patients with maxillofacial trauma from a single Hospital of Tabriz. J. Evolution Med. Dent. Sci. 2016;5(44):2742-2745, DOI: 10.14260/jemds/2016/640

\section{INTRODUCTION}

By increasing the population in the cities, lifestyle change and people have more physical activities like sports and other time-out activities. These changes may lead to increase the rate of injuries, especially maxillofacial fractures because of anatomical features of mandibular and lower face region. ${ }^{(1,2)}$ Different aetiologies of injuries, fracture patterns and various injuries between gender and age groups is associated with different physical activities.(3) Trauma pattern is affected by socioeconomic status, culture of people in different countries.(4-6) The main causes of trauma include Motor Vehicle Accident (MVA).(7) Intimate Partner Violence (IPV), fall, sport and occupational injuries. While expanding the use of safety belt, driver's air bag, controlling driver speed and increasing the violence have changed demographic information about maxillofacial injuries.(8)

Financial or Other, Competing Interest: None.

Submission 14-02-2016, Peer Review 12-05-2016,

Acceptance 17-05-2016, Published 01-06-2016.

Corresponding Author:

Dr. Ali Zarandi,

Assistant Professor,

Department of Periodontics,

Faculty of Dentistry,

Tabriz University of Medical Sciences,

Tabriz, Iran.

E-mail:dr.alizarandi@gmail.com

DOI: $10.14260 /$ jemds/2016/640
The previous report elucidated that $75 \%$ of mandible fractures related to the MVA and IPV, 7\% to job accidents, 7\% to fall and $4 \%$ to sport-related accident.(9) The present research was conducted at the Department of Oral and Maxillofacial Surgery, Emam Reza Hospital, Tabriz University of Medical Sciences, to analyse aetiological factors in line of age and gender in lower face fractures during the one year between 2013 and 2014.

\section{METHODS AND MATERIALS}

This study designed as cross-sectional study. Following Ethics Committee permission, data were collected from patient's documents. Totally, 284 cases with mandible fractures were assessed. The inclusion criteria were lower face fractures, hospitalization in oral and maxillofacial surgery of the Emam Reza Hospital and availability of patient's documents. Also, patient with partial data of fractures were excluded from the study. Finally, 34 cases omitted of the study since data was not completely registered in their documents, but 250 patients had full data. In the questionnaire demographic information of the patients were recorded which include patient code, age, gender, final diagnosis of fractures and cause of trauma. Also, the fractures region in mandible was noted. These locations of fractures were body, symphysis, angle, parasymphyseal, ramus, condylar, subcondylar and coronoid.

Also, another location of face involved in injuries was recorded such as mid-face (Le Fort I and II) and zygoma region. 
The result was analysed by descriptive statistics, frequency and chi-square test in SPSS $21(\mathrm{P}<0.05)$.

\section{RESULTS}

In this study, 250 patients with lower face fractures was assessed that $83.6 \%$ (209 subjects) of patients was male and $16.4 \%$ (41 subjects) was female. Mean of age in the male group was $30.03 \pm 16.57$ years old ( 2 to 113 years old); and in the female group were $30.66 \pm 14.17$ ( 3 to 58 years old). Frequency of the patients according to the age groups is shown in Table 1. The most of fractures happened in 21-30 age groups. At all from the 250 patients, 438 fractures were determined, one fracture in 63 patients (25.01\%), two fractures in 148 subjects (59.37\%), three fractures in 35 patients $(14.06 \%)$ and four fractures in 4 patients $(1.56 \%)$. The most frequency of trauma was seen in subcondylar region (38.28\%) and lowest frequency related to coronoid region (1.56\%) (Table 2). Assessment cause of trauma showed that frequency of fractures attributed to accident (53.9\%), fall (24.2\%), IPV (4.7\%), blunt trauma (11.7\%), sport-related accident $(4.7 \%)$ and falling/blunt trauma (0.8\%) respectively (Table 3). Table 4 dedicated frequency of related factors in trauma according to age groups. The results indicated that in all age groups, the higher frequency was reported from the accident. However, frequency of other cause of trauma was different in age groups that these differences was statistically significant based on the chi-square test. Also, frequency of trauma cause was shown in Table 5 with respect to gender. In both genders the accident was the main aetiological factor, in which the rate of accident was higher in the female patients than the male patients $(71.4 \%$ vs. $50.5 \%)$. The difference between other trauma factors was statistically significant between male and female $(\mathrm{p}<0.05)$.

\begin{tabular}{|c|c|c|}
\hline Age Group & $\mathbf{\%}$ & Number \\
\hline $20<$ & 29.7 & 74 \\
\hline $21-30$ & 32.0 & 80 \\
\hline $31-40$ & 16.4 & 41 \\
\hline $41-50$ & 10.2 & 26 \\
\hline$>50$ & 11.7 & 29 \\
\hline \multicolumn{2}{|c|}{ Table 1: Frequency of the Patients } \\
According to the Age Groups \\
\hline
\end{tabular}

\begin{tabular}{|c|c|c|c|}
\hline Location & $\begin{array}{c}\text { Percentage between } \\
\text { 438 Fractures }\end{array}$ & $\begin{array}{c}\text { Percentage between } \\
\text { 250 Patients }\end{array}$ & Number \\
\hline Body & 19.20 & 33.59 & 54 \\
\hline Symphysis & 13.39 & 23.44 & 33 \\
\hline Parasymphysis & 7.59 & 13.28 & 20 \\
\hline Condylar & 4.46 & 7.81 & 96 \\
\hline Subcondylar & 21.88 & 38.28 & 4 \\
\hline Angle & 19.64 & 34.38 & 13 \\
\hline Coronoid & 0.89 & 1.56 & 36 \\
\hline Ramus & 2.68 & 4.69 & 6 \\
\hline Midface & 8.04 & 14.06 & 2.34 \\
\hline
\end{tabular}

\begin{tabular}{|c|c|c|}
\hline Cause & $\mathbf{0}$ & Number \\
\hline MVA & 53.9 & 135 \\
\hline Fall & 24.2 & 12 \\
\hline IPV & 4.7 & 29 \\
\hline Blunt trauma & 11.7 & 12 \\
\hline Sport-related & 4.7 & 1 \\
\hline Fall/contact & 0.8 & 250 \\
\hline \multicolumn{2}{|c|}{ Table 3: Frequency of Aetiological Factors of Trauma in the Maxillofacial Fractures } \\
\hline
\end{tabular}

\begin{tabular}{|c|c|c|c|c|c|c|c|}
\hline \multirow{2}{*}{ Total } & \multicolumn{5}{|c|}{ Age Group (Years Old) } & & \\
\hline & $>\mathbf{5 0}$ & 41-50 & 31-40 & 21-30 & $<20$ & & \\
\hline 135 & 12 & 16 & 25 & 41 & 41 & Number & \multirow{2}{*}{ (MVA) } \\
\hline $53.9 \%$ & $40.0 \%$ & $61.5 \%$ & $61.9 \%$ & $51.2 \%$ & $55.3 \%$ & $\%$ & \\
\hline 61 & 12 & 10 & 8 & 23 & 8 & Number & \multirow{2}{*}{ Fall } \\
\hline $24.2 \%$ & $40.0 \%$ & $38.5 \%$ & $19.0 \%$ & $29.3 \%$ & $10.5 \%$ & $\%$ & \\
\hline 12 & 4 & & 2 & 6 & & Number & \multirow{2}{*}{ IPV } \\
\hline $4.7 \%$ & $13.3 \%$ & & $4.8 \%$ & $7.3 \%$ & & $\%$ & \\
\hline 29 & 2 & & 4 & 8 & 15 & Number & \multirow{2}{*}{ Blunt trauma } \\
\hline $11.7 \%$ & $6.7 \%$ & & $9.5 \%$ & $9.8 \%$ & $21.1 \%$ & $\%$ & \\
\hline 12 & & & & 2 & 10 & Number & \multirow{2}{*}{ Sport-related } \\
\hline $4.7 \%$ & & & & $2.4 \%$ & $13.2 \%$ & $\%$ & \\
\hline 1 & & & 1 & & & Number & \multirow{2}{*}{ Falling+contact } \\
\hline $.8 \%$ & & & $2.4 \%$ & & & $\%$ & \\
\hline \multicolumn{6}{|c|}{$\chi^{2}=31.16, p=0.046$} & \multicolumn{2}{|c|}{ Chi-Square Tests } \\
\hline
\end{tabular}




\begin{tabular}{|c|c|c|c|c|}
\hline \multirow{2}{*}{ Total } & \multicolumn{2}{|c|}{ Gender } & & \\
\hline & Female & Male & & \\
\hline 135 & 29 & 106 & Number & \multirow{2}{*}{ (MVA) } \\
\hline $53.9 \%$ & $71.4 \%$ & $50.5 \%$ & $\%$ & \\
\hline 61 & 10 & 51 & Number & \multirow{2}{*}{ Fall } \\
\hline $24.2 \%$ & $23.8 \%$ & $24.3 \%$ & $\%$ & \\
\hline 12 & & 12 & Number & \multirow{2}{*}{ IPV } \\
\hline $4.7 \%$ & & $5.6 \%$ & $\%$ & \\
\hline 29 & 2 & 27 & Number & \multirow{2}{*}{ Blunt trauma } \\
\hline $11.7 \%$ & $4.8 \%$ & $13.1 \%$ & $\%$ & \\
\hline 12 & & 12 & Number & \multirow{2}{*}{ Sport-related } \\
\hline $4.7 \%$ & & $5.6 \%$ & $\%$ & \\
\hline 1 & & 1 & Number & \multirow{2}{*}{ Fall+contact } \\
\hline $.8 \%$ & & $.9 \%$ & $\%$ & \\
\hline \multicolumn{3}{|c|}{$\chi^{2}=5.02, p=0.041$} & \multicolumn{2}{|c|}{ Chi-Square Tests } \\
\hline & 5: Compo & ncy of 1 & with Res & \\
\hline
\end{tabular}

\section{DISCUSSION}

According to the results, the male/female ratio involved with lower face fractures was 5:1 in these patients. This is in line with other studies, which showed that male have more injuries in the lower face. $(3,4,10,11)$ Previous literature in India and Austria showed that the frequency of female involved in trauma was higher than other societies, while the male: female ratio was more totally. This is associated with socio-economic activity, violence and other risk factors (Accident, fall, IPV) of male in the different society. $(12,13)$ The most fractures in this study was accrued in the third decade of life (21-30 years old) that was same as other reports.(10),(14),(15) Erol et al, found that further fractures were happening in 11-20 years old group.(16) In the present study the level of trauma in groups $>50$ years old was increased, but was lower than other groups. This fact may be attributable to physiological symptoms of aging, disease and increasing the self-care in this group. The most patients had two fractures and permanent fracture pattern was symphysis and parasymphysis linked with subcondylar on the one side of the face because of injuries and anatomical position of the lower jaw. The most fractures in the lower jaw related to subcondylar, angle and body, respectively. In this study, other part of the face in fractures was mid-face and zygomatic region.

The previous reports about epidemiological study of lower face fractures are different. So, condylar.(10) body.(4) symphysis.(17) ramus, body and parasymphysis were determined as the prevalence position in fractures. A result confirmed that the accident is an important trauma factor (Accident by bus, motorcycle), which was same as early information.(4),(8,10) According to other studies in Nigeria, Libya, America and Europe, accidents were known as the main cause of facial fractures. Also, in some data demonstrated that by changing culture in cities IPV is key reason and IPV in male group and accident in female group was described as trauma factors.(3) Falling was the second cause of injuries in this study, then blunt trauma and IPV was identified as fracture issues. It is considerable that blunt trauma was not recognized as an independent risk factor for fractures in previous studies. On the other hand, the patients hide the fact of IPV because of legal and ethical outcome and especially with family problems or abuse and alcohol use.(8) Investigation of the trauma factor with respect to age showed that the accident was the first cause of mandible fracture that was changed in $40-61 \%$ of age groups. In age group less than 20 years blunt trauma was second factor, while in another age group falling was reported as the second trauma factor. Also in this group and 41-50 years age, IPV was not recorded in documents. In contrast with these results, Hoppe et al found that IPV was as a prevalence factor on fracture.(3) Overall, IPV identified as the third main cause of trauma. $(3,10,18)$ Frequency of trauma by contact with blunt particle in the third and fourth decade of life was higher in age $>50$ years old $(9.8,9.5 \%$ vs. $6.7 \%)$. Finally, sport-related fractures were seen in the age group less than 30 years old. This may be related to social-cultural factor of the sport in our country. The search for causes of trauma according to gender showed that in both male and female, the accident was the main factor in trauma. Accident in female $(71.4 \%)$ was more in comparison with male $(50.5 \%)$. But according to the data of female's documentation, no report of IPV and sport-linked injuries was found. While, 5.6\% IPV and 5.6\% sport-related injuries were determined in the male. In both genders, fall had approximately the same rate and blunt trauma was high in the male in contrast with the female $(13.1 \%$ vs. $4.8 \%)$, that this may be associated with the type of male's activities in occupational position.

\section{CONCLUSION}

The most patients with lower face fractures were seen in the male and third decade of their life. The main cause of fracture in all age groups and both genders was MVA and then fall, blunt trauma, IPV and sport-related accidents respectively. Probably, gender and age have a significant effect on the frequency of epidemiology of trauma, that in this study mandible fractures by reason of IPV and sport was not reported in the females. In addition, fracture in subcondylar region was known as the most involved part of trauma in the lower face region and most patients had more than one fracture in the face.

\section{REFERENCES}

1. Zix JA, Schaller B, Lieger O, et al. Incidence, aetiology and pattern of mandibular fractures in central Switzerland. Swiss medical weekly 2011;141:w13207.

2. Dongas P, Hall GM. Mandibular fracture patterns in Tasmania, Australia. Australian dental journal 2002;47(2):131-7.

3. Hoppe IC, Kordahi AM, Paik AM, et al. Age and sex-related differences in 431 pediatric facial fractures at a level 1 trauma center. Journal of craniomaxillofa surg 2014;42(7):1408-11. 
4. Samieirad S, Tohidi E, Shahidi-Payam A, et al. Retrospective study maxillofacial fractures epidemiology and treatment plans in southeast of Iran. Med oral patol oral cir bucal 2015;20(6):e729-36.

5. Kumar GB, Dhupar V, Akkara F, et al. Patterns of maxillofacial fractures in Goa. Journal of maxillofacial and oral surgery 2015;14(2):138-41.

6. Yoffe T, Shohat I, Shoshani Y, et al. Aetiology of maxillofacial trauma-a 10-year survey at the chaim sheba medical center, tel-hashomer. Harefuah 2008;147(3):192-6,280.

7. Bowley D, Boffard K. Patterns of injury in motor vehicle accidents. World Wide Wounds 2002.

8. Goulart DR, Colombo Ldo A, De Moraes M, et al. What is expected from a facial trauma caused by violence? Journal of oral \& maxillofacial research 2014;5(4):e4.

9. Saini P. Oral and maxillofacial trauma. British dental journal 2013;214(9):480.

10. Ghodke MH, Bhoyar SC, Shah SV. Prevalence of mandibular fractures reported at C.S.M.S.S dental college, aurangabad from february 2008 to september 2009. Journal of International Society of Preventive \& Community Dentistry 2013;3(2):51-8.

11. Bali R, Sharma P, Garg A, et al. A comprehensive study on maxillofacial trauma conducted in Yamunanagar, India. Journal of injury \& violence research 2013;5(2):108-16.

12. Subhashraj K, Nandakumar N, Ravindran C. Review of maxillofacial injuries in Chennai, India: a study of 2748 cases. British journal of oral \& maxillofacial surgery 2007;45(8):637-9.
13. Van Beek GJ, Merkx CA. Changes in the pattern of fractures of the maxillofacial skeleton. International journal of oral and maxillofacial surgery 1999;28(6):424-8.

14. Motamedi MH, Dadgar E, Ebrahimi A, et al. Pattern of maxillofacial fractures: a 5-year analysis of 8,818 patients. Journal of trauma and acute care surgery 2014;77(4):6304.

15. Patrocínio LG, Patrocínio JA, Borba BHC, et al. Mandibular fracture: analysis of 293 patients treated in the hospital of clinics, federal university of uberlândia. Braz J Otorhinolaryngol 2005;71(5):560-5.

16. Erol B, Tanrikulu R, Gorgun B. Maxillofacial fractures. analysis of demographic distribution and treatment in 2901 patients (25-year experience). Journal of craniomaxillo-facial surgery 2004;32(5):308-13.

17. Jung HW, Lee BS, Kwon YD, et al. Retrospective clinical study of mandible fractures. Journal of the Korean Association of Oral and Maxillofacial Surgeons 2014;40(1):21-6.

18. Malara P, Malara B, Drugacz J. Characteristics of maxillofacial injuries resulting from road traffic accidents--a 5 year review of the case records from department of maxillofacial surgery in katowice, poland. Head \& face medicine 2006;2:27. 